\title{
A novel strategy to accurately represent the carrier gas properties of droplets evaporating in a combustion environment
}

\section{Citation for published version (APA):}

Sacomano Filho, F. L., Krieger Filho, G. C., van Oijen, J. A., Sadiki, A., \& Janicka, J. (2019). A novel strategy to accurately represent the carrier gas properties of droplets evaporating in a combustion environment. International Journal of Heat and Mass Transfer, 137, 1141-1153.

https://doi.org/10.1016/j.ijheatmasstransfer.2019.03.164

DOI:

10.1016/j.ijheatmasstransfer.2019.03.164

Document status and date:

Published: 01/07/2019

\section{Document Version:}

Accepted manuscript including changes made at the peer-review stage

\section{Please check the document version of this publication:}

- A submitted manuscript is the version of the article upon submission and before peer-review. There can be important differences between the submitted version and the official published version of record. People interested in the research are advised to contact the author for the final version of the publication, or visit the DOI to the publisher's website.

- The final author version and the galley proof are versions of the publication after peer review.

- The final published version features the final layout of the paper including the volume, issue and page numbers.

Link to publication

\footnotetext{
General rights

- You may freely distribute the URL identifying the publication in the public portal. follow below link for the End User Agreement:

www.tue.nl/taverne

\section{Take down policy}

If you believe that this document breaches copyright please contact us at:

openaccess@tue.nl

providing details and we will investigate your claim.
}

Copyright and moral rights for the publications made accessible in the public portal are retained by the authors and/or other copyright owners and it is a condition of accessing publications that users recognise and abide by the legal requirements associated with these rights.

- Users may download and print one copy of any publication from the public portal for the purpose of private study or research.

- You may not further distribute the material or use it for any profit-making activity or commercial gain

If the publication is distributed under the terms of Article $25 \mathrm{fa}$ of the Dutch Copyright Act, indicated by the "Taverne" license above, please 


\title{
A novel strategy to accurately represent the carrier gas properties of droplets evaporating in a combustion environment
}

\author{
Fernando Luiz Sacomano Filho, ${ }^{\mathrm{a}, \mathrm{b}, *}$, Guenther Carlos Krieger Filho ${ }^{\mathrm{a}}$, Jeroen Adrianus \\ van Oijen ${ }^{\mathrm{c}}$, Amsini Sadiki ${ }^{\mathrm{b}}$, Johannes Janicka ${ }^{\mathrm{b}}$ \\ ${ }^{a}$ Laboratory of Environmental and Thermal Engineering, Universidade de São Paulo, São Paulo, Brazil. \\ ${ }^{b}$ Institute for Energy and Power Plant Technology, Technische Universität Darmstadt, Darmstadt, Germany. \\ ${ }^{c}$ Department of Mechanical Engineering, Technische Universiteit Eindhoven, Eindhoven, Netherlands.
}

\begin{abstract}
A novel approach to accurately represent the carrier gas thermo-chemical properties of droplets evaporating in combustion environment is proposed and validated in the context of reduced chemistry approaches. Numerical analyses of two of the most employed droplet evaporation models in computational fluid dynamics applications and their subsequent impact on combustion processes are also performed. The study comprehends a systematic investigation of both models and different procedures used to address the relevant thermo-chemical properties for the evaporation modeling. Initially, investigations are addressed in a single droplet framework. Herein, available experimental data give support to the corresponding discussions. In a second part, both selected models and the simplification using air as carrier gas mixture are investigated in the context of flames propagating in droplets mists. A detailed chemistry model is used to represent the combustion of ethanol in air. A simplification strategy is subsequently investigated in terms of the relevant thermo-chemical properties for the evaporation modeling. The resulting strategy is successfully validated in a numerical context for flames propagating in droplet mists. Results aim to help the choice of methods employed for droplet evaporation modeling in a more general context. The methodology adopted in our analyses allows the assessment of each simplification and converges to an optimal combination of the studied methods.
\end{abstract}

Keywords: Droplet evaporation, Evaporation modeling, Ethanol, Spray combustion, Droplet mists

\section{Introduction}

Droplets evaporating in combustion environment often experience various mixture compositions at different temperatures during their lifetime [1-5]. Since combustion

\footnotetext{
${ }^{*}$ Corresponding author

Email address: fernando.sacomano@usp.br (Fernando Luiz Sacomano Filho)
} 
reaction may involve hundreds of chemical species, a detailed description of the mixture in CFD (Computational Fluid Dynamics) simulations is a challenging task. Usually, detailed chemistry information is not available and simplifications become indispensable to address the carrier gas properties (e.g. [2, 6-10]). Such an issue can be intensified when sub-grid scale aspects of the properties estimation, and the droplet evaporation modeling itself, are considered. In fact, droplet evaporation modeling is a recurrent topic in CFD simulations of spray flames [1, 2, 6, 10-14]. The large disparity of scales involving heat and mass exchanges on droplets and the others related to the flow dynamics makes the evaporation a sub grid phenomenon [15]. Therefore, the computation of these exchange mechanisms is a matter of modeling.

Reviews (e.g. [1, 16, 17]) and recent studies (e.g. [11, 14]) can be found in the literature covering many topics related to the droplet evaporation modeling. Miller et al. [16] compare eight evaporation models in a quite objective form for single-droplet evaporation tests. Sazhin [17] presents a more comprehensive discussion about different aspects regarding the subject, while keeping the focus on CFD implementations. Yet Sirignano [1] covers a great part of the droplet evaporation modeling, starting from the fundamentals up to complex and specific application issues. Chrigui et al. [11] and Noh et al. [14] present comparisons among different models in turbulent flows. Chrigui et al. [11] investigated two of the most used evaporation models in spray combustion simulations in non-reactive acetone sprays, namely the infinite liquid conductivity model proposed by Abramzon and Sirignano [18] and the non-equilibrium approach by Miller et al. [16]. In this study, the non-equilibrium model of Miller et al. [16] showed better agreement with experimental data. Both models have been analyzed together with the classic rapid-mixing model (see [16]) by Noh et al. [14] in non-reactive and reactive sprays of $n$-heptane. No significant difference has been reported between both Abramzon and Sirignano [18] and Miller et al. [16] models. However, these two approaches demonstrated to deliver better results than the classic rapid-mixing model.

A common characteristic of most of the previously discussed works is the simplification of properties calculation. For example, the vapor pressure is often computed with the Clausius-Clapeyron equation (e.g. [5, 6, 11, 12, 16, 18,-20]). A preceding work of the present one (see [15]) shows that simplifications in calculation of properties like the vapor pressure may lead to wrong evaporation rates in common situations found in spray combustion. Therein, both models analyzed by Chrigui et al. [11] and Noh et al. [14] are investigated for flames propagating in droplets mists, which offer a more isolated scenario for the assessment of evaporation modeling. Differently, from the results obtained in [14], the models of Abramzon and Sirignano [18] and Miller et al. [16] show significant differences between each other. Perhaps, the turbulent flow existing in [14] homogenizes the modeling differences observed for laminar flows in [15].

Preliminary analyses also conducted in [15] show that simplifications of the mixture composition to represent the carrier gas are capable to interfere not only with the evaporation rates, but also with the prediction of flame propagation speeds. Approaches that account for detailed mixture composition of the carrier gas can also be found in [4, 10, 12, 21]. Those presented by Franzelli et al. [4], Ma et al. [12], and Both [10] are developed in the context of tabulated chemistry, while the work of Sierra Sanchez [21] has no restrictions to chemistry modeling techniques. Regarding the treatment given to 
the droplet evaporation modeling, the work of Both [10] can be seen as a continuation of the study presented in [12]. Motivated by some computational issues originated from the high memory requirements to store detailed chemistry information ${ }^{1}$ in [12], Both [10] shows a low memory cost alternative to consider such a detailed chemistry information. There, the surrounding gas mixture is expressed in terms of controlling variables of the applied chemistry database. The efficient usage of computational resources related to the determination of surrounding gas properties was also the motivation of [21] to propose a simplification based on fixed Schmidt and Prandtl numbers. Particularly, this technique is employed by Franzelli et al. [4] in combination with a reduced number of species to compute droplet evaporation in combustion environments.

It is the purpose of this work to propose a feasible approach for an accurate representation of the carrier gas thermo-chemical properties of droplets evaporating in combustion environment. The focus is on general reduced chemistry calculations, that include for example the Flamelet Generated Manifolds (FGM) ${ }^{2}$ method. Different from the approaches adopted in [4, 10, 12, 21], the presented one is exclusively based on the construction of combinations of small number of chemical species that can accurately recover the properties of the actual carrier mixture. Particularly, this work extends, deepens, and addresses opened issues of the preliminary studies presented in [15] in a systematic form. As done in [11, 14, 15], the performance of the infinite liquid conductivity models proposed by Abramzon and Sirignano [18] and Miller et al. [16] are assessed in different scenarios and for different fuels. However, analysis in combustion environment are reserved for ethanol combustion, in which the influence of evaporation models and the proposed approach to address the carrier gas properties are conducted in one-dimensional flames propagating in droplet mists. Ethanol reaction with air is represented by the detailed chemistry mechanism proposed by Marinov [23]. It represents the oxidation of ethanol in air by means of 57 species and 379 intermediate reactions. The investigation of the two selected evaporation models in combustion environment aims to evaluate the sensitivity of both to the correct representation of the carrier mixture. Notice that, it is not the purpose of such an investigation to indicate a best model for combustion simulations.

The remaining of this manuscript is divided into three parts. Firstly, a summary of the employed modeling techniques, as well as the proposed strategy to improve the flame speed calculations caused by the insufficient representation of the mixture composition are described. In the second part, results are presented and discussed in four subsections. Initially, single droplet analyses are performed in a non-reacting atmospheres, in which experimental data is available. In the next subsection, the two selected evaporation models are compared in the framework of one-dimensional flames propagating in droplets mists of ethanol including a detailed description of the chemistry. Afterwards, the proposed methodology is evaluated concerning the thermo-

\footnotetext{
${ }^{1}$ Both [10] indicates that the use of the detailed composition in FGM tables leads to huge memory requirements that limit the size of the applicable reaction mechanisms, limits parallelization and has a substantial performance penalty.

${ }^{2}$ Observe that, in principle, all information about the chemistry reactions can be stored in a FGM database. However, this is usually not done. Many times, only a few species are stored to reduce memory load (e.g. 17, 10, 11 22]).
} 
chemical properties used to address the evaporation modeling. This methodology is validated in the sequel with flames propagating in droplet mists. Finally, the third and last part summarizes the main outcomes and closes this manuscript with some final remarks and an outlook.

\section{Modeling Techniques}

An Eulerian-Lagrangian approach is applied to numerically describe flames propagating in droplet mists, whereas single droplet computations are conducted in an isolated scheme. In this last scenario, the carrier gas properties are maintained constant at (when not explicitly specified in the text) experimentally defined conditions. Yet for the reactive cases, a full inter-phase two-way coupling is considered. In both cases, computations are performed with the CFD code CHEM1D [24], which has been extended with a Lagrangian module in [13].

In order to isolate the diverse aspects of the spray combustion, the simulations presented in this manuscript mimic unstrained laminar flames propagating in monodispersed and isotropic droplet mists. Similar to Neophytou and Mastorakos [25], the following simplifications and approximations are assumed: (a) the multi-dimensional aspects of the droplets dispersion does not modify the flame surface, (b) mists are diluted, thereby no droplets interaction are accounted for, (c) no micro-mixing model is included, so that all the mass evaporated from a drop fills instantaneously the host control volume ${ }^{3}$. Furthermore, parcels are tracked instead of real drops. In this way, a parcel may represent more or even less than one real drop. The role played by them reduces to a dispersed source of fuel which follows the physical models described in the two following sections. For more details about the numerical methods and simplifications applied to the dispersed phase the reader is referred to [5].

\subsection{Liquid Phase}

In the one-dimensional Lagrangian framework, the tracking of a parcel is essentially described by two ordinary differential equations (ODE) (e.g. Sirignano [1])

$$
\frac{d x_{p}}{d t}=u_{p}, \quad \text { and } \quad \frac{d u_{p}}{d t}=\frac{3}{4} \frac{C_{D}}{d_{p}} \frac{\rho}{\rho_{p}}\left|u-u_{p}\right|\left(u-u_{p}\right)
$$

for the parcel position and acceleration, respectively. In the present context, drag is the unique force acting on a droplet. For both equations, the subscript $p$ indicates quantities of droplet parcels. Specifically, $x_{p}$ corresponds to the droplet position, $\rho$ is the density, $u$ the velocity, $t$ is time, and $d_{p}$ the droplet diameter. To compute the drag coefficient $C_{D}$ the model proposed by Yuen and Chen [26] is adopted.

Heat and mass exchanges are respectively described by

$$
\frac{d T_{p}}{d t}=\frac{f_{2} N u}{3 \operatorname{Pr}}\left(\frac{\theta_{1}}{\tau_{p}}\right)\left(T-T_{p}\right)+\left(\frac{L_{V}}{c_{l}}\right) \frac{\dot{m}_{p}}{m_{p}}, \text { and }
$$

\footnotetext{
${ }^{3}$ When a droplet crosses a cell boundary a splitting factor is used to interpolate the source of vapor between the two host control volumes.
} 


$$
\frac{d m_{p}}{d t}=-\frac{S h}{3 S c}\left(\frac{m_{p}}{\tau_{p}}\right) H_{M},
$$

with $T$ the temperature, $N u$ the Nusselt number, $f_{2}$ a correction factor due to evaporation (see Table 11, $\operatorname{Pr}$ the Prandtl number, $L_{V}$ the heat of vaporization, $\theta_{1}$ is a ratio of specific heat (see Table 1,$c_{l}$ the specific heat of the liquid, $\tau_{p}=\rho_{p} d_{p}^{2} / 18 \mu$ expresses the particle relaxation time and $\dot{m}_{p}=d m_{p} / d t$. In Eq. 3. $S h$ is the Sherwood number, $S c$ the gas Schmidt number ${ }^{4}$ and $H_{M}$ represents the specific driving potential for mass transfer (see Table 1). Observe that heat transfer by radiation is not considered.

Table 1: Specific variables of the selected models

\begin{tabular}{lccc}
\hline Model & $f_{2}$ & $H_{M}$ & $\theta_{1}$ \\
\hline M1 & $\frac{\beta}{e^{\beta}-1}$ & $\ln \left[1+B_{M, n e q}\right]$ & $\frac{c_{p, G}}{c_{l}}$ \\
M2 & $\frac{-\dot{m}_{p}}{m_{p} B_{T}}\left[\frac{3 \tau_{p} P r}{N u}\right]$ & $\ln \left[1+B_{M, e q}\right]$ & $\frac{c_{p, V}}{c_{l}}$ \\
\hline
\end{tabular}

Table 2: Used references for gas properties

\begin{tabular}{lcc}
\hline Property & Air & $\mathrm{N}_{2}$ \\
\hline Dynamic viscosity $-\mu$ & {$[27]$} & {$[28]$} \\
Thermal conductivity $-\lambda$ & {$[27]$} & {$[28]$} \\
Specific sensible heat $-c_{p}$ & {$[29]$} & {$[28]$} \\
Binary diffusion coef. of vapor in gas $-D_{i j}$ & {$[28]$} & {$[28]$} \\
\hline
\end{tabular}

The structures of Eqs. 2 and 3 follow those proposed by Miller et al. [16], which simplify the switch among different modeling approaches. Two of them are selected for the present investigations. One is the infinite liquid conductivity version of the non-equilibrium evaporation model of Miller et al. [16], which is denoted hereafter as M1. The other is the Abramzon-Sirignano model (see [18]), which is identified by M2 throughout this manuscript. This choice is based on the fact that both have been broadly applied in different studies about spray combustion (e.g. [11, 14, 15, 20]) due to their ability to represent droplet evaporation though considering infinite liquid thermal conductivity. According to the structure of Eqs. 2 and 3 both models differ by the expression for $f_{2}, H_{M}$, and $\theta_{1}$. Table 1 specifies each one of these quantities for both models. It is important to mention that, great part of the notation used in [16] is also employed here to make a clear connection with that review paper, where different aspects of both modeling approaches are comprehensively discussed.

\footnotetext{
${ }^{4}$ The non-dimensional numbers $P r$ and $S c$ are explicitly computed at each time integration of the evaporation equations based on material properties of the mixture film surrounding each tracked droplet. Notice that both are also used to compute the $L e=S c / P r$, which is consequently employed for instance in Eq. 8 When unity $L e$ is assumed for the evaporation, $S c=P r$.
} 
In Table 1. $c_{p}$ the specific heat at constant pressure, $B_{T}$ and $B_{M}$ denote the Spalding transfer numbers for heat and mass, respectively. Subscripts ${ }_{G}$ and ${ }_{V}$ correspond to surrounding gas properties and vapor properties, while $e q$ and neq to properties evaluated with the assumption of phase equilibrium and considering effects of non-equilibrium, respectively.

Considering the strategy proposed in Miller et al. [16], the following methods are used to obtain the surrounding gas properties. The first consists of the definition of reference values for the temperature and vapor mass fraction which are used to evaluate gas and vapor material properties in the surrounding gas zone. Herein, the well known " $1 / 3$ rule" "see [26]) is applied. Accordingly, both reference values are given by the linear combination of the surface value (weighted by $2 / 3$ ) and of the far away (carrier gas) value (weighted by 1/3). Recent works [6, 12] highlight the importance of the usage of the $1 / 3$ rule, which is therefore no further analyzed here. Once that both reference values are defined, two procedures are used to calculate the mixture properties at the gas film. The semi-empirical Wilke rule is adopted for the computation of $\mu$ and $\lambda$, while the specific heat is computed by

$$
c_{p, G}=Y_{R} c_{p, V}+\left(1-Y_{R}\right) c_{p, \infty},
$$

where $Y_{R}$ is the reference vapor mass fraction and $c_{p, \infty}$ is the specific heat of the carrier gas. Observe that according to this procedure, the carrier gas properties must be recomputed at the reference temperature. Hence, the carrier mixture composition is preserved while properties are recalculated following the methods presented in [24]. 5] For more details about the mixing averaging applied here, the reader is referred to [16].

It is discussed in the further sections that the carrier gas is usually simplified as air. In this situation, air properties are obtained from the databases listed in Table 2 . It is important to highlight that, gas density is obtained by the ideal gas law. A property that receives more attention here is the vapor pressure at the droplet surface. With this quantity, the mass fraction of vapor on the droplet surface $Y_{s}$ is computed, which is consequently used to estimate $B_{M}$ as follows

$$
B_{M}=\frac{Y_{s}-Y_{\infty}}{1-Y_{s}} .
$$

In the equation above, $Y_{s}$ is straightly obtained from the denoted equilibrium molar fraction $X_{s, e q}$ (ratio of the vapor and the ambient pressure) in model M2, and it is connected with the non-dimensional evaporation parameter $\beta$ in model M1 as

$$
X_{s, n e q}=X_{s, e q}-\beta\left(\frac{2 L_{K}}{d_{p}}\right),
$$

where

$$
\beta=-\left(\frac{3 \tau_{p} P r}{2}\right) \frac{\dot{m}_{p}}{m_{p}} \text { and } L_{K}=\frac{\mu \sqrt{2 \pi T_{p} R / M_{V}}}{\alpha_{e} S c P}
$$

\footnotetext{
${ }^{5}$ In applications involving the FGM method, the proposed procedure implies that only the carrier mixture composition is utilized. Therefore, there is no need to store the necessary properties for the evaporation modeling in a FGM manifold.
} 
in which $L_{K}$ is the Knudsen layer thickness, $M_{V}$ the molar mass of vapor, $R$ the universal ideal gas constant, $P$ the ambient pressure, and $\alpha_{e}$ the molecular accommodation coefficient. As suggested by [16], this last coefficient is assumed equal to unity.

The heat transfer Spalding number can be written as function of $B_{M}$ as given by

$$
B_{T}=\left(1+B_{M}\right)^{\phi}-1 \quad \text { with } \quad \phi=\frac{c_{p, v}}{c_{l}} \frac{S h^{*}}{N u^{*}} \frac{1}{L e} .
$$

$B_{T}$ is an explicit variable in model M2, in which effects of the Stefan flow are considered in the boundary layer thickness in terms of the modified Nusselt and Sherwood numbers as follows

$$
\begin{aligned}
& N u^{*}=2+\frac{N u-2}{F_{T}}, \quad F_{T}=\frac{\left(1+B_{T}\right)^{0.7}}{B_{T}} \ln \left(1+B_{T}\right), \\
& S h^{*}=2+\frac{S h-2}{F_{M}}, \quad F_{M}=\frac{\left(1+B_{M}\right)^{0.7}}{B_{M}} \ln \left(1+B_{M}\right) .
\end{aligned}
$$

In summary, both models differ from each other in three main aspects. As indicated in Table 1 model M2 considers the vapor specific heat in $\theta_{1}$ instead of the surrounding gas specific heat as in M1. This is a direct outcome of the hypotheses assumed on the derivation of heat transfer equations from the differential form of the energy equation (see [1, 17]). Another clear difference between both models is that model M2 assumes phase equilibrium at the droplet surface, while model M1 accounts for nonequilibrium effects (see [16]). Finally, the third main aspect refers to the consideration of the film thickness on the computation of Nusselt and Sherwood numbers in model M2 (see [18]), which is not considered in model M1. Besides these conceptual differences, some others occur in the solution algorithm of each model. Iterative solution procedures are implemented in different parts of each model (see [16]). However, such algorithm differences do not produce significant deviations between calculation results. Except for these differences, both models are similar. Namely, both are derived from steady evaporation assumption for spherically symmetric droplets based on the infinite liquid conductivity approach and constant gas properties at the film (see [16, 17]). For more detailed information about the differences listed above, the reader is referred to the review paper of Miller et al. [16].

\subsection{Gas Phase}

The description of the carrier phase follows a variable-density low Mach number formulation. According to the strategy presented by Somers [24], the set of equations used to compute steady freely propagating flat flames in CHEM1D is

$$
\begin{gathered}
\frac{\partial \dot{m}}{\partial x}=S_{\mathrm{V}}^{L}, \\
\frac{\partial\left(\dot{m} Y_{i}\right)}{\partial x}-\frac{\partial}{\partial x}\left(\frac{\lambda}{L e_{i} c_{p}} \frac{\partial Y_{i}}{\partial x}\right)=\dot{\omega}_{i}+\delta_{i k} S_{\mathrm{V}}^{L}, \quad \text { and }
\end{gathered}
$$




$$
\frac{\partial(\dot{m} h)}{\partial x}-\frac{\partial}{\partial x}\left(\frac{\lambda}{c_{p}} \frac{\partial h}{\partial x}\right)=\frac{\partial}{\partial x}\left(\frac{\lambda}{c_{p}} \sum_{i=1}^{N_{s}}\left(\frac{1}{L e_{i}}-1\right) h_{i} \frac{\partial Y_{i}}{\partial x}\right)+S_{\mathrm{h}}^{L} .
$$

Equation 11 ensures the mass conservation of the coupled system, where $\dot{m}=\rho u$ is the mixture mass flux, $x$ the spatial coordinate, and $S_{\mathrm{V}}^{L}$ the source term of vapor. The conservation of chemical species is described by Eq. 12 Herein, $Y_{i}$ is the mass fraction of the species $i \in\left[1, N_{s}-1\right], N_{s}$ total number of participating species, $\lambda$ the thermal conductivity, $c_{p}$ the isobaric specific heat, $L e_{i}$ the Lewis number for species $i$, $\dot{\omega}_{i}$ the reaction rate for species $i$, and $\delta_{i k}$ the Kronecker delta. To account for differential diffusion aspects, $L e_{i}$ is obtained by the Mixture Averaged ${ }^{6}$ approach. The subscript $k$ in Eq. 12 refers to the vapor species. The last equation of this set is associated to the conservation of energy, which is expressed in terms of the absolute enthalpy $h$. The absolute enthalpy of each transported species is represented by $h_{i}$, while the coupling term between phases is denoted by $S_{\mathrm{h}}^{L}$. For details about the coupling source terms, the reader is referred to [5, 13].

An important aspect is that the carrier phase is described in a steady-state formulation, while the dispersed phase follows an unsteady framework. To couple both approaches, the methodology proposed in [5] is applied here.

The chemical mechanism proposed by Marinov [23] is employed to address the combustion reactions in a detailed chemistry context. It represents the oxidation of ethanol in air by means of 57 species and 379 intermediate reactions.

\subsection{Simplification of the actual mixture composition}

Droplets interacting with reacting flows may experience various compositions of the carrier gas, as the concentration of chemical species (apart from vapor and oxidizer) significantly changes according to the reaction progress. In section 3 , it is presented that the composition of the carrier gas does not only interfere with the evaporation process itself, but also with the speed of flames propagating in droplet mists. In view of modeling accuracy, it is of interest to characterize the mixture composition with high fidelity. Nevertheless, this can be many times prohibitive, since a detailed description of the chemistry is computationally costly. Because of necessary simplifications in reduced mechanisms or limited storage in tabulated chemistry methods, this detailed description is usually not available during the computational time. Commonly, carrier gas properties are simplified as air properties at the same temperature and pressure of the mixture (e.g. [2, 6, 9$]$ ). In order to improve the mixture representation for evaporating droplets, a procedure is proposed here under the perspective of reduced chemistry methods.

Two strategies have been evaluated to accurately represent the effects of the mixture composition on the evaporation process. However, both are delineated by the same concept: the search for a minimal combination of major species that could represent the relevant mixture properties to the evaporation process (those listed in Table 2). In the first strategy, the actual molar fraction of air $X_{\text {air }}\left(X_{\text {air }}=X_{\mathrm{N}_{2}}+X_{\mathrm{O}_{2}}\right)$ and fuel $X_{\text {fuel }}$ are

\footnotetext{
${ }^{6}$ Hirschfelder-Curtiss approximation (see [30]
} 
kept as their actual values, whereas the combined molar fraction of other species equals to $X_{\text {major }}=1-\left(X_{\text {air }}+X_{\text {fuel }}\right)$. According to this method, information about molar or mass fractions of $\mathrm{O}_{2}$ and $\mathrm{N}_{2}$ must be available during the performance of a simulation. In the second strategy, just the actual molar fractions of fuel and the fractions needed in the major species definition are necessary, the air molar fraction is estimated by $X_{\text {air }}=1-\left(X_{\text {major }}+X_{\text {fuel }}\right)$. Hereafter, the first strategy is denoted as species diluted (SD), while the second as air diluted (AD). Notice that in the SD framework, the combination of molar or mass fractions of major species and fuel allows to determine the mass fraction of air, while in $\mathrm{AD}$ scenario explicit data of $\mathrm{N}_{2}$ and $\mathrm{O}_{2}$ are necessary. In the reduced chemistry approach such data are always available (at least easily achievable), whereas in the FGM context it must be ensured that two data columns are reserved for $\mathrm{N}_{2}$ and $\mathrm{O}_{2}$ in the database.

A common need of both described strategies is however the definition of species combinations. On one hand, the number of species shall be as small as possible. On the other hand, the accuracy of the properties representation must be sufficient. Herein, we arbitrary sought for a combination formed by a maximum of 10 species. To define a valid combination, we arbitrary set a maximum allowed property $(\psi)$ deviation

$$
\varepsilon_{i}=100 \% \times\left|\psi_{\text {mix }}-\psi_{\text {major, } \mathrm{i}}\right| / \psi_{\text {mix }}
$$

of 5\%. In Eq. 14 the subscript ${ }_{\text {mix }}$ refers to properties values computed with the actual mixture, while major to those computed with the major species combination $i$.

The ten major species chosen to compose our different combinations correspond to those at which droplets are exposed for a longer time interval when reaction starts. To find these species, the following procedure has been applied: (1) definition of the exposure time as $\Delta t_{\text {exp }}\left(z_{\text {over }}\right)=l_{p}\left(z_{\text {over }}\right) / s_{l, s}\left(z_{\text {over }}\right)$, where $l_{p}\left(z_{\text {over }}\right)$ is the penetration length 7 for a droplet in a mist with overall mixture fraction $z_{\text {over }}{ }^{8}$, and $s_{l, s}\left(z_{\text {over }}\right)$ is the corresponding laminar flame speed; (2) calculation of the averaged mixture composition $\overline{Y_{k, z_{\text {over }}}}$ throughout the penetration length for a given overall mixture fraction; (3) sum of the different species averaged mass fractions over a range of overall mixture fractions weighted by the exposure time, namely $\overline{Y_{s, k}}=\sum_{z_{\text {over }}} \overline{Y_{k, z_{\text {over }}}} \cdot \Delta t_{\mathrm{exp}}\left(z_{\mathrm{over}}\right)$, with $z_{\text {over }}$ ranging from 0.070 to 0.220 in a constant step of 0.001 ; and (4) determination of the ten most higher values of $\overline{Y_{s, k}}$ and the species corresponding to them. In the present work, the solution of flames propagating in droplets mists with: initial droplet diameter $d_{p, 0}=75 \mu \mathrm{m}$, injection position of $l_{\text {inj }}=-3.0 \mathrm{~cm}$, computed with the evaporation model M2 and the thermo-chemical properties using the actual mixture composition; is used in such a selection procedure.

Figure 1 illustrates the evolution of the normalized values of $\overline{Y_{k, z \text { ove }}} 9$ of the ten selected major species, i.e. $k=[1,2, \ldots, 10]$, throughout the range $z_{\text {over }} \in[0.070,0.220]$. The numeric indexes of the selected major species are listed in Table 3 The variation

\footnotetext{
${ }^{7}$ Distance from the position where the combustion reactions start to the one where the droplet disappears. Herein, the position where reactions start is chosen as the cell center in which the temperature gradient is positive after the droplet injection.

${ }^{8} z_{\text {over }}$ is achieved when all the liquid is completely pre-vaporized.

${ }^{9}$ Normalization is performed using the maximum encountered value of $\overline{Y_{k, z_{\text {over }}}}$.
} 

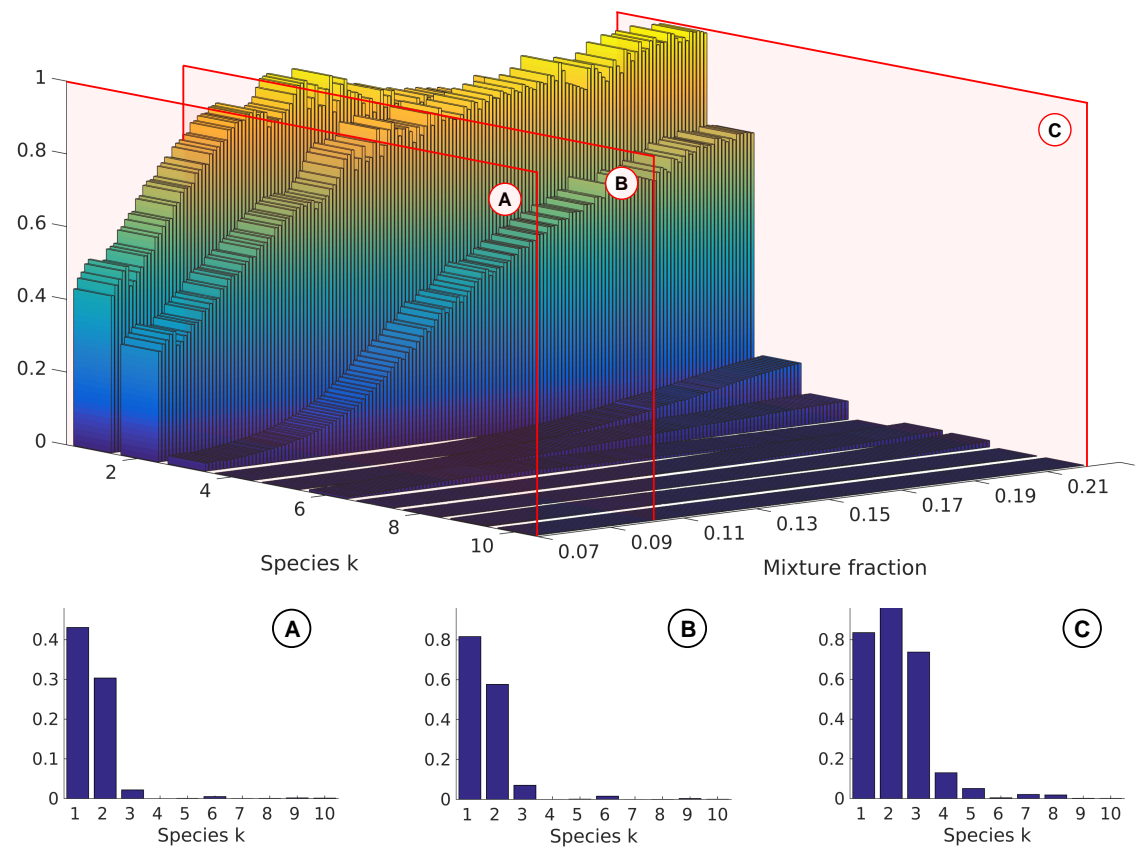

Figure 1: Normalized species mass fractions $\overline{Y_{k, z \text { over }}}$ for different overall mixture fraction values. Slices $\mathbf{A}$, $\mathbf{B}$, and $\mathbf{C}$ have the respective overall mixture fraction values: $0.070,0.100$, and 0.220 .

of $\overline{Y_{k, z_{\text {zover }}}}$ values in a function of $z_{\text {over }}$ (see Fig. 1) is the reason that the average throughout the mixture fraction range has been applied for the search procedure. Three bar plots labeled with the letters A, B, and C are included in Fig. 1 to help the visualization of maximum values of $\overline{Y_{k, z_{\text {over }}}}$ in lean, stoichiometric, and rich overall mixture compositions, respectively. An example of the $\overline{Y_{k, z \text { over }}}$ variation depending on the composition can be clearly seen by means of $\overline{Y_{3, z_{\text {zover }}}}(\mathrm{CO})$. Its value gradually increases with the overall mixture fraction.

Details of the choice of major species combinations, as well as their corresponding assessment on properties calculation, are presented in section 3.3

\section{Results and Discussion}

Results are presented in four parts. In a first one, the relevance of different evaporation modeling features is assessed in a single droplet setup. Yet, the influence of evaporation models and the simplification of the carrier mixture as pure air is evaluated in a second part. After that, the different simplification strategies of the carrier gas mixture are assessed using the relevant thermo-chemical properties to the evaporation.

\footnotetext{
${ }^{10}$ The chemical species $\mathrm{O}$ should not be interpreted as the diatomic oxygen $\mathrm{O}_{2}$, which is present in air. The species $\mathrm{O}$ is formed during the evolution of combustion reactions and has been found as one of the ten major species following the selection procedure presented in this section.
} 
Table 3: Indexes of selected major species

\begin{tabular}{lcc}
\hline Index $(\mathrm{k})$ & Species name & Species formula \\
\hline 1 & carbon dioxide & $\mathrm{CO}_{2}$ \\
2 & water & $\mathrm{H}_{2} \mathrm{O}$ \\
3 & carbon monoxide & $\mathrm{CO}$ \\
4 & acetylene & $\mathrm{C}_{2} \mathrm{H}_{2}$ \\
5 & hydrogen & $\mathrm{H}_{2}$ \\
6 & hydroxide & $\mathrm{OH}$ \\
7 & methane & $\mathrm{CH}_{4}$ \\
8 & ethylene & $\mathrm{C}_{2} \mathrm{H}_{4}$ \\
9 & oxygen aton & $\mathrm{O}$ \\
10 & acetaldehyde & $\mathrm{CH}_{3} \mathrm{HCO}$ \\
\hline
\end{tabular}

Finally, the impact of these simplifications are analyzed in terms of the speed of flames propagating in droplet mists.

\subsection{Single droplet evaporation analyses}

The decay of $d_{p}^{2}$ along time is presented in Fig. 2 illustrates the performance of both selected models for different operating conditions. Herein, the experimental data obtained by Saharin et al. [31] are used as a benchmark. They correspond to diameter measurements sampled for suspended droplets in $\mathrm{N}_{2}$ quiescent atmospheres with relative humidity of approximately $4 \%$ at four different operating conditions, which are summarized in Table 4

Both models can reasonably well describe the variations in initial droplet diameter and carrier gas temperature. Invariably to all tested conditions, model M2 approaches slightly better to the experimental data. Considering the last stage of the evaporation process, a variation of evaporation rates can be clearly identified in all experimental curves. Recent studies conducted at the University of São Paulo (not presented here) show that this change originates from the multi-component liquid solution. The used anhydrous ethanol has a composition of $99.5 \%$ of ethanol and $0.5 \%$ water. Despite being an azeotrope, i.e. solution that cannot be separated by distillation (see for instance [1]), the combination of the liquid water with the relative humidity causes this well defined second slope in $d_{p}^{2}$ curves. Furthermore, studies performed by Saharin [32] shows that by increasing the amount of water in the fuel the inflexion of $d_{p}^{2}$ curves becomes more evident.

Table 4: Operating conditions for ethanol single droplet evaporation

\begin{tabular}{lcc}
\hline Case & $T[\mathrm{~K}]$ & $d_{p, 0}[\mu \mathrm{m}]$ \\
\hline 1 & 293 & 651 \\
2 & 353 & 628 \\
3 & 473 & 609 \\
4 & 673 & 430 \\
\hline
\end{tabular}




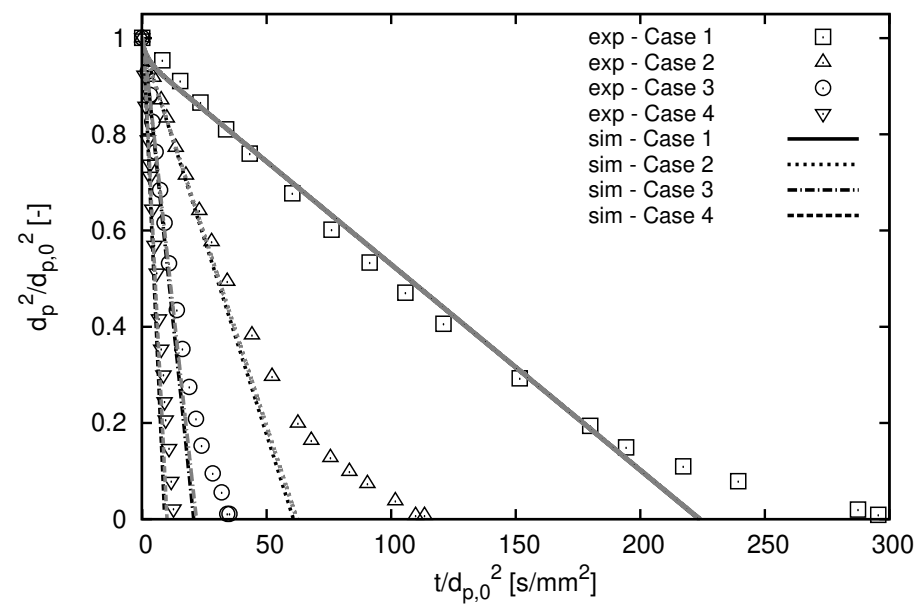

Figure 2: Performance of both selected models (M1 and M2) for ethanol droplets at different initial conditions. Black - M1 data, Gray - M2 data.

Simulations presented in Fig. 2 have been conducted with the vapor pressure model based on the Wagner equation (see [33]). As shown in Fig. 3, this model shows better results for a broad range of gas temperatures when compared to selected Antoine and Clausius-Clapeyron equations. During the development of this study, attention has been given to the relevance of accurate prediction of the vapor pressure (VP) on the droplet surface. Specifically, the vapor pressure at the droplet surface is used to estimate the mass transfer number $\left(B_{M}\right.$ - see discussion in section 2.1 in various evaporation models [1, 16, 17]. The different calculation methods for the vapor pressure are listed in Table 5. Different procedures are found in the literature to compute this quantity. Herein, five of them (see Table 5) are chosen and evaluated. The simulations presented for this analysis have been performed with the model M2.

Table 5: Description of vapor-pressure labels.

\begin{tabular}{ll}
\hline Model & Label \\
\hline Clausius-Clapeyron as in [16] & VP1 \\
Clausius-Clapeyron based on $P_{c}$ (see [33]) & VP2 \\
Wagner equation (see [33]) & VP3 \\
Antoine equation (see [33]) & VP4 \\
Antoine equation from NIST Web-book & VP5 \\
\hline
\end{tabular}

Figure 3 shows the effect of different vapor pressure models on droplet evaporation for the two most extreme operating conditions listed in Table 4. Differences between models are evident in the low temperature scenario, while all of them deliver similar results for the high temperature case. In both scenarios, Wagner and Antoine equation 
models behave quite similar since these are based on experimental data fitting. As mentioned by Poling et al. [33], Clausius-Clapeyron methods are supposed to introduce considerable errors on vapor pressure calculations for temperatures far from the one used as reference. Herein, the boiling point temperature of ethanol at $1.0 \mathrm{~atm}(351.5 \mathrm{~K})$ is used for VP1 and VP2. Considering that the gas temperature is much higher than the boiling point temperature, it is not surprising that both Clausius-Clapeyron methods are not far from the other three VP models in high temperature cases. Therefore, care must be taken when using this method to estimate the mass transfer number $B_{M}$ at low gas temperatures.
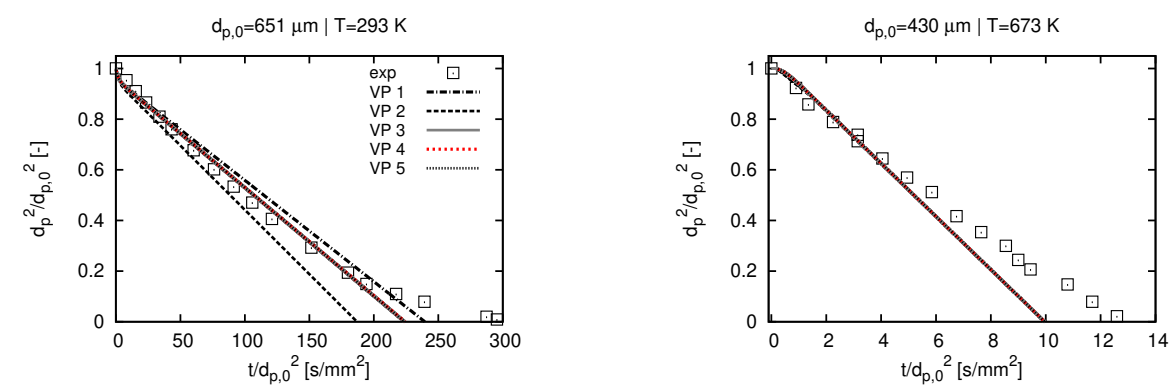

Figure 3: Effect of different vapor pressure models on the evolution of droplet evaporation for low (left) and high (right) ambient temperatures.

The assumption that $S c=P r$ which implies $L e=1$ is investigated here. This assumption is sometimes adopted to simplify properties calculations (see e.g. [16]). However, such an assumption modifies the relationship between heat and mass transfer numbers $\left(B_{T}\right.$ and $\left.B_{M}\right)$ as well as between $N u$ and $S h$. Hereafter, analyses of ethanol evaporation are restricted to case 4 of Table 4 since the high gas temperature better represents the atmospheres found in reacting cases, which is our final object of study.

Figure 4 shows that the $L e=1$ assumption does not strongly interfere with the evaporation modeling. This assumption slightly increases the evaporation rate for model M2. Effects are more pronounced for forced convection cases extracted from [16]. Figure 5 shows $d_{p}^{2}$ curves for an hexane case at moderate gas temperature and high convection and decane at high temperature and moderate convection. By assuming $L e=1$ higher evaporation rates are obtained in the simulations of the hexane droplet, while not so pronounced effects are seen for the decane droplets case. This outcome indicates that influences are more evident for $\mathrm{Nu}$ and $S h$ than for the relationship between $B_{T}$ and $B_{M}$. Regarding the performance of models M1 and M2 in forced convection scenarios, model M1 slightly better approaches the experimental data in the hexane case, while both show similar deviation from the experimental data in the decane case. In this last scenario, the evaporation rate predicted with model M2 agrees better with the experimental measurements than that obtained with model M1.

The last part of this section is dedicated to the evaluation of the simplification of the actual mixture by air in a single droplet setup. To perform it, properties listed in Table 2 are extracted from thermal and transport data provided in Marinov's mechanism [23]. Both property computation methods (i.e. from Table 2 and those using Marinov's 


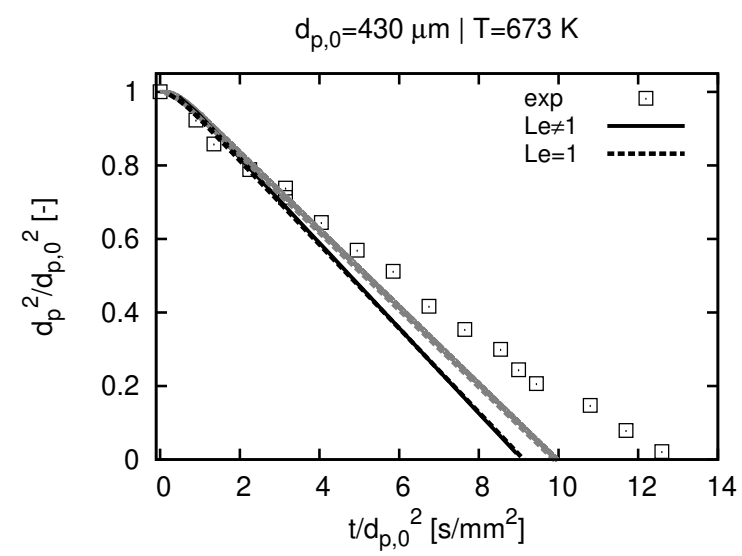

Figure 4: Influence of Le for ethanol. Black - M1 data, Gray - M2 data.
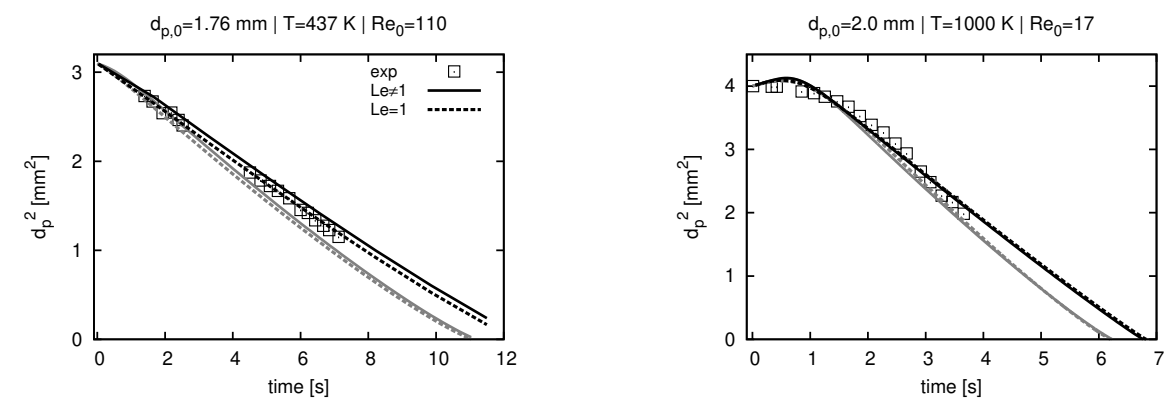

Figure 5: Influence of Le simplification in forced convection atmosphere for hexane (left) and decane (right). Black - M1 data, Gray - M2 data. Operating conditions of both cases are found on the top of each corresponding sub-figure. Experimental results of hexane (left) are from Downingm [34], while those of decane (right) are from Wong and Lin 35

mechanism) are validated before advanced simulations. Hence, computations of $d_{p}^{2}$ decay assuming a mixture of pure air are also presented in Fig. 6. Results clearly demonstrate that the usage of properties listed in Table 2 (Fig. 6. M2 air data) and the ones based on data provided by Marinov's mechanism (Fig. 6. M2 air code) agree quite good to each other when assuming pure air as carrier gas. Observe that a case computed with $\mathrm{N}_{2}$ is included as reference (Fig. 6. $\mathrm{N}_{2}$ data) and simulations are only conducted with model M2 to keep the analysis concise. Bearing in mind that a great part of air is $\mathrm{N}_{2}$, it is not surprising that no significant changes occur when $\mathrm{N}_{2}$ is substituted by air. The results presented in Fig. 6for air demonstrate that the computations based on data provided by Marinov's mechanism are correctly performed, which allow the usage of this method for further investigations with other mixture compositions.

To evaluate the influence of the detailed mixture composition in a single-droplet setup, $\mathrm{N}_{2}$ is substituted by a mixture of burnt products (quite close to the chemical 


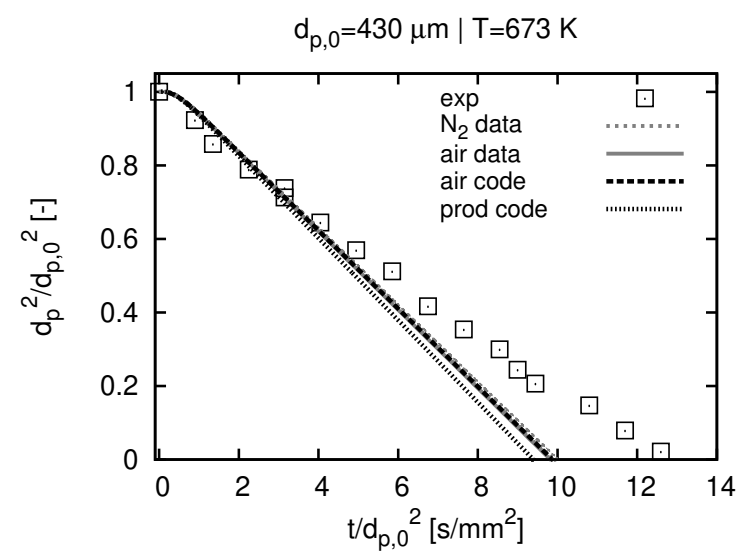

Figure 6: Analysis of gas composition. In the labels, "data"refers to properties computed from the references indicated in Table 2 while "code"to those obtained from the Marinov's mechanism [23].

equilibrium) obtained from an adiabatic freely propagating gaseous flame at stoichiometric conditions. The mixture temperature is the same as for case 4 of Table 4 to make both cases comparable. Results are included in Fig. 6 and show that the evaporation rate is increased by approximately $5 \%$. This indicates that assuming actual mixture properties to compute evaporation in combustion cases may influence the flame characteristics. To analyze this, simulations of freely propagating flames in droplets mists are addressed in the next part.

\subsection{Influence of the evaporation modeling on flames propagating in droplets mists}

The flame propagation speed is a simple and useful variable that can indicate the role played by the evaporation modeling and the simplification of the gas mixture in combustion processes. Since some turbulent spray combustion models do consider the laminar flame speed as an input [e.g. the Artificially Thickened Flame (ATF) model [7, [8], the behavior of different models and simplifications investigated here are expected to be an important factor at least in turbulent combustion of lean mixtures and those close to stoichiometry.

Figure 7 shows the behavior of the spray flame speed $s_{l, s}$ for mists with different droplet initial diameters $d_{p, 0}$ and overall equivalence ratios $\phi_{\text {over }}$. For most flames simulated here, except when explicitly mentioned in the text, droplets are injected at the same position $l_{\text {inj }}$ (i.e. $3.0 \mathrm{~cm}$ upstream of the reaction zone). In the two overall equivalence ratio scenarios, $d_{p, 0}$ varies from $25 \mu \mathrm{m}$ to $75 \mu \mathrm{m}$. Both models M1 and M2 are compared together with the two different representations of the mixture, i.e. actual mixture composition and its simplification by air. This figure exhibits that the choice of evaporation modeling clearly has impact on the flame behavior. Overall, model M1 delivers faster flames than those computed with model M2. Deviations vary according to $d_{p, 0}$ in both scenarios, where their values increase with $d_{p, 0}$. Specifically, the smallest values found for $\varepsilon$ in $\phi_{\text {over }}=2.3$ occurs due to the approach of M1 computations 
to the lower flammability limit found by reducing $d_{p,}, 11$, which approximates M1 to M2 computations. In all computed flames, deviations between models do not cross the limit of $7 \%$.
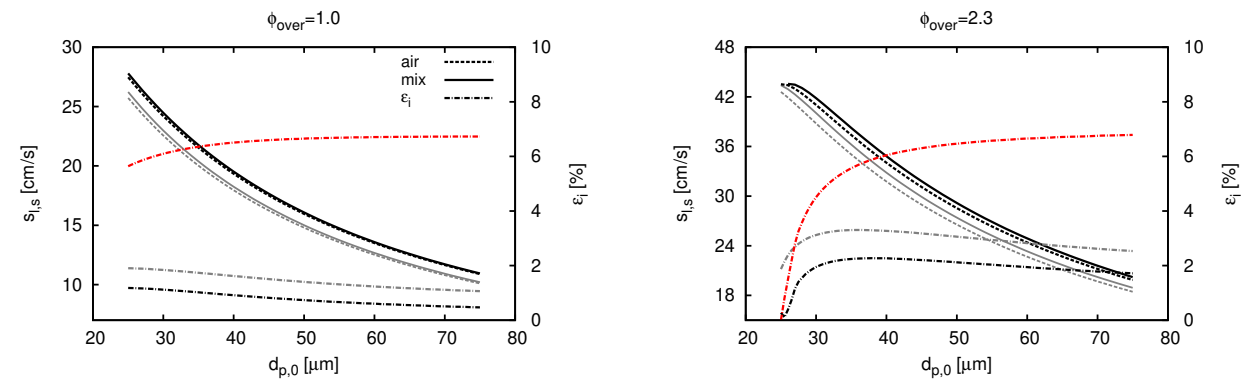

Figure 7: $s_{l, s}$ in function of $d_{p, 0}$ for a stoichiometric $\left(\phi_{\text {over }}=1.0\right.$ on the left $)$ and a rich $\left(\phi_{\text {over }}=2.3\right.$ on the right) droplet mist. Black - M1 data, Gray - M2 data, Red - deviation between models M1 and M2.

A similar maximum deviation value is also observed in Fig. 8 showing dependence of flame propagation speed on overall equivalence ratio. Nevertheless, the smooth variation of $\varepsilon$ throughout $d_{p, 0}$ in Fig. 7/is not observed by increasing $\phi_{\text {over }}$ while keeping $d_{p, 0}$ constant. Here, $\varepsilon$ clearly peaks for a $\phi_{\text {over }}$ value between 1.0 and 1.5 in both initial droplet sizes. After this maximum, $\varepsilon$ reduces for rich mixtures down to the value of 0.0 . Specifically at this point, a strong inflection of the $\varepsilon$ curve occurs because of the usage of the absolute value operator in Eq. 14. For richer mixtures, deviations start to grow again. Another important aspect noticed in these plots is that not only values of $s_{l, s}$ differ between models for a given $\phi_{\text {over }}$, but also the resulting flammability limits vary in terms of $\phi_{\text {over }}$. Computations with model M2 show broader flammability limits than model M1. Such variations on computed flammability limits may explain the grow of deviation after the point where $\varepsilon=0$.

The influence of the droplets injection distance to the flame is also investigated here. Figure 9 shows that by reducing $l_{\text {inj }}$ deviations between models turn higher. It is important to highlight here that as the residence time in the pre-vaporization zone reduces, droplets reach the reaction zone with larger diameters and are presumably farther from the equilibrium with the carrier gas. The higher deviation values found for larger droplets reinforce this explanation. In contrast to the previous plots, deviations cross the limit of $8 \%$ for the case $d_{p, 0}=75 \mu \mathrm{m}$.

With respect to the simplification of the detailed mixture composition by air, differences do exist in Figs. 7, 8, and 9 but are not so pronounced as deviations observed between both evaporation models. In all cases, model M2 demonstrates to be more sensitive to this simplification as M1.

Plots presented in Fig. 7 show that deviations caused by the simplification of the detailed mixture composition by air approximately double by changing the overall equiv-

\footnotetext{
[5]

${ }^{11}$ Detailed discussions about flammability limits in droplet mists can be found in Sacomano Filho et al.
} 

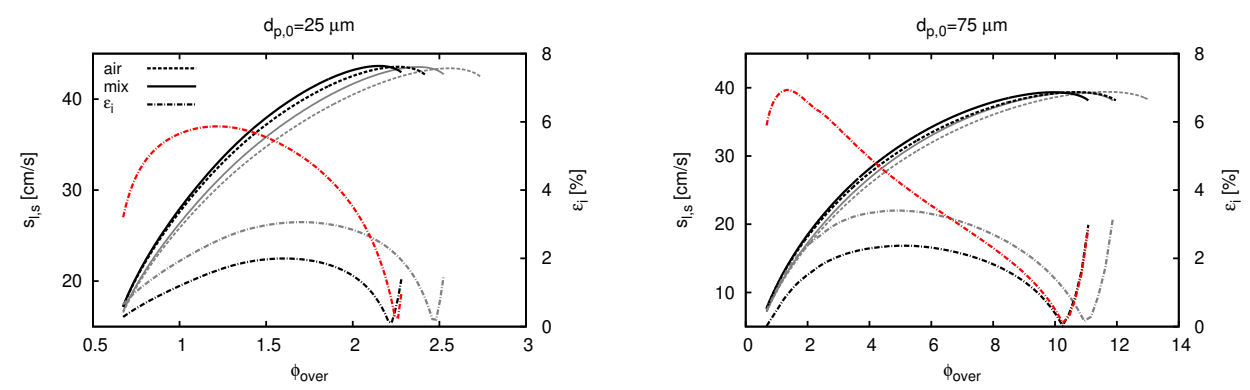

Figure 8: $s_{l, s}$ in function of $\phi_{\text {over }}$ for droplet mists of $d_{p, 0}=25 \mu \mathrm{m}$ (left) and $d_{p, 0}=75 \mu \mathrm{m}$ (right). Black M1 data, Gray - M2 data, Red - deviation between models M1 and M2.
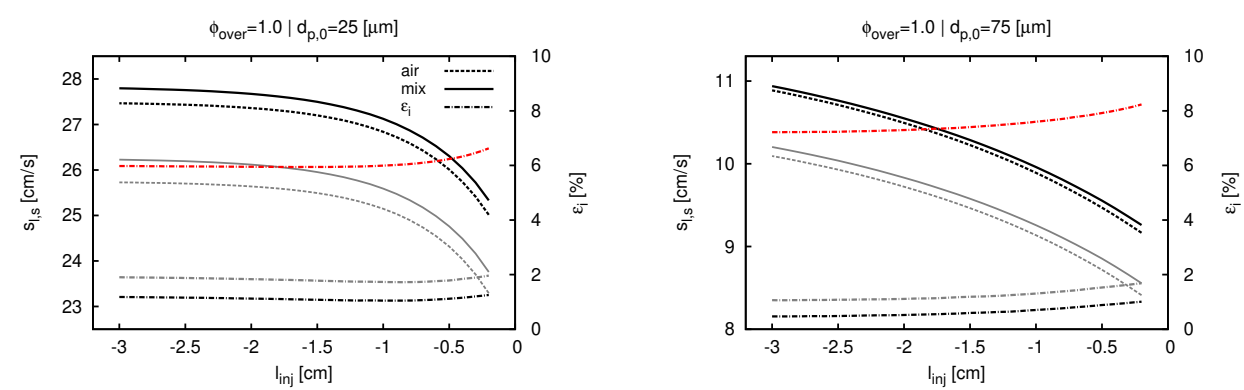

Figure 9: $s_{l, s}$ in function of $l_{\text {inj }}$ for stoichiometric droplet mists of $d_{p, 0}=25 \mu \mathrm{m}$ (left) and $d_{p, 0}=75 \mu \mathrm{m}$ (right). Black - M1 data, Gray - M2 data, Red - deviation between models M1 and M2.

alence ratio from stoichiometric to rich for both models. Nevertheless, the trend of $\varepsilon$ points out that deviations do not increase with $d_{p, 0}$ (ranging from $25 \mu \mathrm{m}$ to $75 \mu \mathrm{m}$ ) by maintaining $\phi_{\text {over }}$ constant as mentioned in [15]. Also in this sense, the usage of $\varepsilon$ combined with the broader ranges of $\phi_{\text {over }}$ presented in Fig. 8 (when compared to the previous study) shows that the interpretation of the increased deviations with $\phi_{\text {over }}$ while keeping $d_{p, 0}$ constant would not hold. The corresponding values for $\varepsilon$ are not monotonic and show a maximum (in the investigated $\phi_{\text {over }}$ range) in the region of $\phi_{\text {over }} \in[1.5,2.0]$ for $d_{p, 0}=25 \mu \mathrm{m}$ and $\phi_{\text {over }} \in[4.0,6.0]$ for $d_{p, 0}=75 \mu \mathrm{m}$. Such observations show that some care must be taken when using only $\varepsilon$ on the interpretation of models behavior.

Indeed, $\varepsilon$ can indicate how far a target variable is from a reference solution. Specific to this section, the target variable is the laminar flame speed and the reference cases are always based on computations considering the actual mixture composition on the evaporation modeling. Nevertheless, it must be highlighted that using only $\varepsilon$ presents limitations on the interpretation of many deviations encountered in previous plots. For instance, the reduction of $\varepsilon$ values as $\phi_{\text {over }}$ increases while keeping $d_{p, 0}$ constant indicates deviations only in values of $s_{l, s}$. In fact, the longer exposure of droplets to the burnt products (that occurs when $\phi_{\text {over }}$ increases) intensifies the differences between cases with different mixture composition approaches. The contrasting 
flammability limits highlight it.

The flammability limits encountered for instance when $\phi_{\text {over }}$ increases while keeping $d_{p, 0}$ constant can be explained by the concentrated heat removal of the mixture caused by droplets evaporation, as comprehensively discussed in [5]. According to the evaporation rate, which is the main influenced aspect in our parametric studies, the heat removal can be intensified or attenuated. In cases when the actual mixture composition is employed, the evaporation rate increases (see Fig. 6) which leads to lower values of $\phi_{\text {over }}$ for the upper flammability limit than those found when the mixture is simplified as air. Similar behavior is also seen when model M1 is compared with model M2 (see Figs. 2 and 4 which illustrates the conditions found in the analyzed combustion cases). In this case, model M1 shows higher evaporation rates throughout the flame, which consequently result in lower flammability limits than those obtained with model M2.

Regarding the maximum deviations (caused by the actual mixture simplification by air) seen in Fig. 8, higher values are found for the scenario where $d_{p, 0}=75 \mu \mathrm{m}$ than for that where $d_{p, 0}=25 \mu \mathrm{m}$. This can be explained by the longer exposure of droplets to the burnt products, where the composition of the actual mixture considerably differs from pure air. A similar explanation can be used to justify the increase in $\varepsilon$ in Fig. 9 . As closer droplets are injected to the flame front, longer they flight through the reaction and burnt products zones.

\subsection{Representation of the mixture by major species}

The two strategies proposed in section 2.3 to simplify the detailed mixture composition in terms of major species, namely the species-diluted SD and air-diluted AD methods, are evaluated here. For that, the four relevant gas properties to the evaporation process, that are listed in table 2 , are used as natural targets. Altogether, eight combinations of major species obtained with the selection procedure presented in section 2.3 for each strategy are compared with actual properties values and those computed with pure air at the same temperature and pressure of the actual mixture. These combinations are presented in Table 6 .

Table 6: Combinations of major species

\begin{tabular}{ll}
\hline Label & Species combination \\
\hline $\mathrm{C} 1$ & $\mathrm{CO}_{2}$ \\
$\mathrm{C} 2$ & $\mathrm{H}_{2} \mathrm{O}$ \\
$\mathrm{C} 3$ & $\mathrm{CO}_{2}+\mathrm{H}_{2} \mathrm{O}$ \\
$\mathrm{C} 4$ & $\mathrm{CO}_{2}+\mathrm{H}_{2} \mathrm{O}+\mathrm{CO}$ \\
C5 & $\mathrm{CO}_{2}+\mathrm{H}_{2} \mathrm{O}+\mathrm{CO}+\mathrm{H}_{2}$ \\
C6 & $\mathrm{CO}_{2}+\mathrm{H}_{2} \mathrm{O}+\mathrm{CO}+\mathrm{H}_{2}+\mathrm{C}_{2} \mathrm{H}_{2}+\mathrm{OH}$ \\
C7 & $\mathrm{CO}_{2}+\mathrm{H}_{2} \mathrm{O}+\mathrm{CO}+\mathrm{H}_{2}+\mathrm{C}_{2} \mathrm{H}_{2}+\mathrm{OH}+\mathrm{CH}_{4}+\mathrm{C}_{2} \mathrm{H}_{4}$ \\
$\mathrm{C} 8$ & $\mathrm{CO}_{2}+\mathrm{H}_{2} \mathrm{O}+\mathrm{CO}+\mathrm{H}_{2}+\mathrm{C}_{2} \mathrm{H}_{2}+\mathrm{OH}+\mathrm{CH}_{4}+\mathrm{C}_{2} \mathrm{H}_{4}+\mathrm{O}+\mathrm{CH}_{3} \mathrm{HCO}$ \\
\hline
\end{tabular}

The number of species gradually increases along with the combinations presented in Table 6 In almost all cases, the combination grows according to the order of the major species listed in Table 3, i.e. from higher to lower concentrations. The only exception occurs for the advance from the combination $\mathrm{C} 4$ to $\mathrm{C} 5$, where $\mathrm{H}_{2}$ is added 
instead of $\mathrm{C}_{2} \mathrm{H}_{2}$. This choice has been taken due to the higher importance of hydrogen when compared with acetylene for the properties investigated. It could be observed that, the addition of $\mathrm{H}_{2}$ to $\mathrm{C} 4$ leads to a more effective combination of four species than using $\mathrm{C}_{2} \mathrm{H}_{2}$ instead.

The comparisons plots presented in Figs. 10, 11, 12, and 13 respectively corresponds to the evolution of the dynamic viscosity $\mu$, thermal conductivity $\lambda$, specific heat at constant pressure $c_{p}$, and binary diffusion coefficient of fuel into the mixture $D_{F, m i x}$. Results are extracted from simulations conducted with a droplet mist formed by ethanol droplets with $d_{p, 0}=75 \mu \mathrm{m}$ at $300 \mathrm{~K}$ introduced in an air stream at $300 \mathrm{~K}$ in a distance of $3.0 \mathrm{~cm}$ upstream the flame front. The evaporation is computed with the model M2 (see section 2.1). The overall mixture fraction $z_{\text {over }}$ is 0.220 which corresponds to an overall equivalence ratio $\phi_{\text {over }}$ of 2.51 . This condition is used as reference since it presents longer penetration times than the leaner mists presented in Fig. 1 . Particularly, the behaviors observed in this section are also seen for leaner mixtures, but less pronounced due to the shorter droplet exposure periods to the combustion products. Following the premises assumed for the major species selection in section 2.3 , the evaluation is conducted here only through the penetration length.
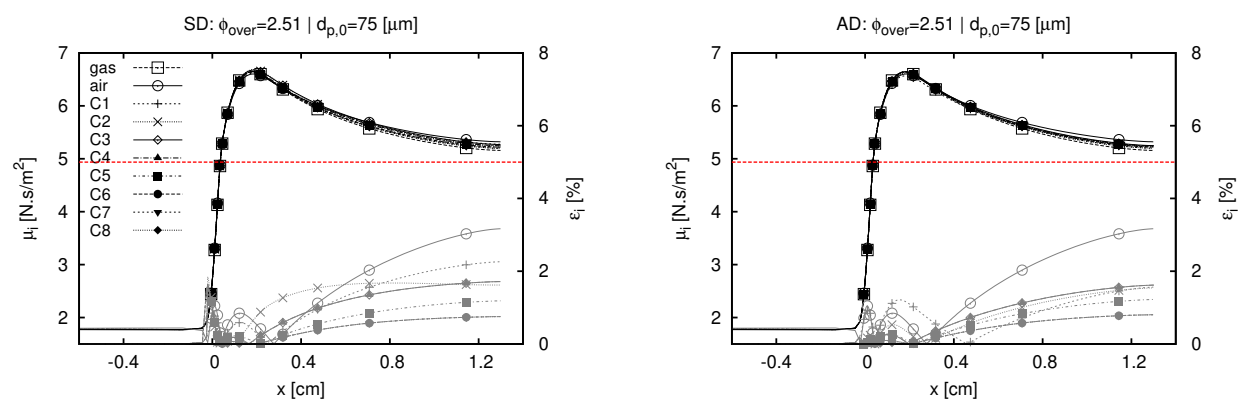

Figure 10: Dynamic viscosity $\mu_{i}$ computations ( $i$ refers to each approach) for the gas film throughout the droplet penetration for a rich mixture $\left(\phi_{\text {over }}=2.51\right)$. Black - $\mu_{i}$, Gray - $\varepsilon_{i}$, Red - $5 \%$ deviation line.

No significant differences among the eight combinations can be observed for the gaseous dynamic viscosity in Fig. 10 for both strategies SD and AD. This is specially not surprising for $\mathrm{AD}$ cases, since property values derived from air at the same temperature and pressure of the actual mixture approaches quite well to the reference data $\left(\varepsilon_{i}<4 \%\right)$. The good approximation of SD values to the reference case demonstrates that combustion products values do not significantly differ from those obtained from air.

The overall behavior noticed for $\mu$ considerably differs in thermal conductivity plots presented in Fig. 11. Thermal conductivities derived from air show deviations close to $40 \%$ of the actual mixture values in Fig. 11. Therefore, the influence of the major species combination and the dilution strategy are more pronounced for this property. Considering the SD results at first, the increase of the species number in a combination allows better estimations of $\lambda$ up to C5. Results obtained with the combinations C5$\mathrm{C} 8$ are quite close to each other and no considerable improvement can be seen by incrementing the combination C5. Despite showing higher deviations than the SD 

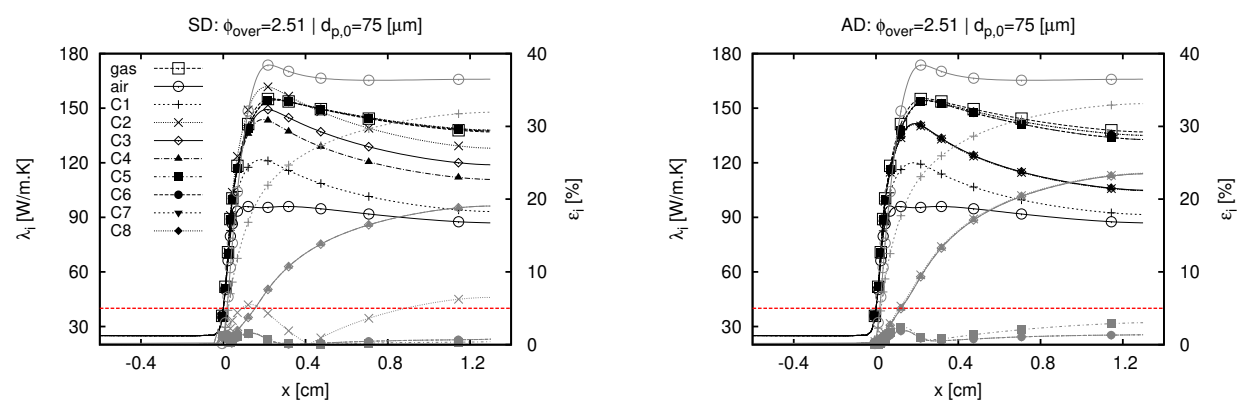

Figure 11: Thermal conductivity $\lambda_{i}$ computations ( $i$ refers to each approach) for the gas film throughout the droplet penetration for a rich mixture $\left(\phi_{\text {over }}=2.51\right)$. Black $-\lambda_{i}$, Gray $-\varepsilon_{i}$, Red $-5 \%$ deviation line.

computation, $\mathrm{AD}$ combination $\mathrm{C} 5$ is also a suitable approach in the present assessment (deviations below 5\%). Differently from SD cases, a gradual improvement is observed by increasing the species number in a combination. Particularly, combinations C2-C4 shows quite similar results.
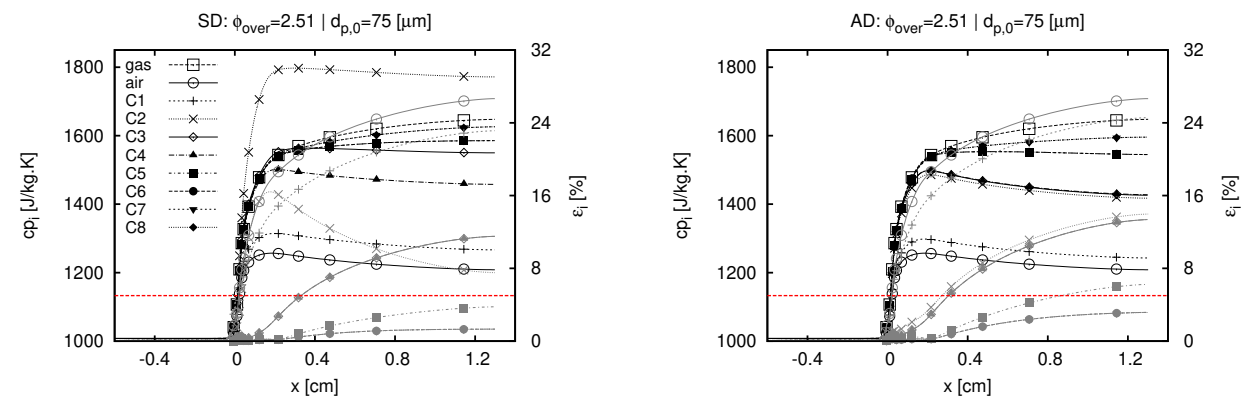

Figure 12: Specific sensible heat at constant pressure $c_{p}$ computations for the gas film throughout the droplet penetration for a rich mixture $\left(\phi_{\mathrm{over}}=2.51\right)$. Black $-c_{p}$, Gray $-\varepsilon_{i}$, Red $-5 \%$ deviation line.

Strong differences among combinations and dilutions are also noticed for the specific heat in Fig. 12. Again, property values derived from air are not accurate approaches of actual mixture values. Deviations reach values above $25 \%$. The contribution of individual species in $c_{p}$ is well pronounced. Values obtained with $\mathrm{CO}_{2}(\mathrm{C} 1)$ and $\mathrm{H}_{2} \mathrm{O}(\mathrm{C} 2)$ are clearly different, whereas their combination (C3) significantly contribute to the approximation to the actual mixture in $\mathrm{SD}$ cases. Considering $\mathrm{AD}$ results, $\mathrm{H}_{2} \mathrm{O}$ (C2) shows the higher individual contribution among the other chosen species. As for the thermal conductivity, the combination C5 delivers deviations below 5\% for SD calculations, while such a criterion is only obtained for C6 in AD cases. Specifically, the increase of the number of species in both dilution methods evolves up to C6, where no significant differences are seen for $\mathrm{C} 7$ and $\mathrm{C} 8$.

Computations of the binary diffusion coefficient of fuel in the gas mixture are presented in Fig. 13. Similar as the results obtained for the dynamic viscosity, values derived from air are not so far from those of the actual mixture. Deviations appear 

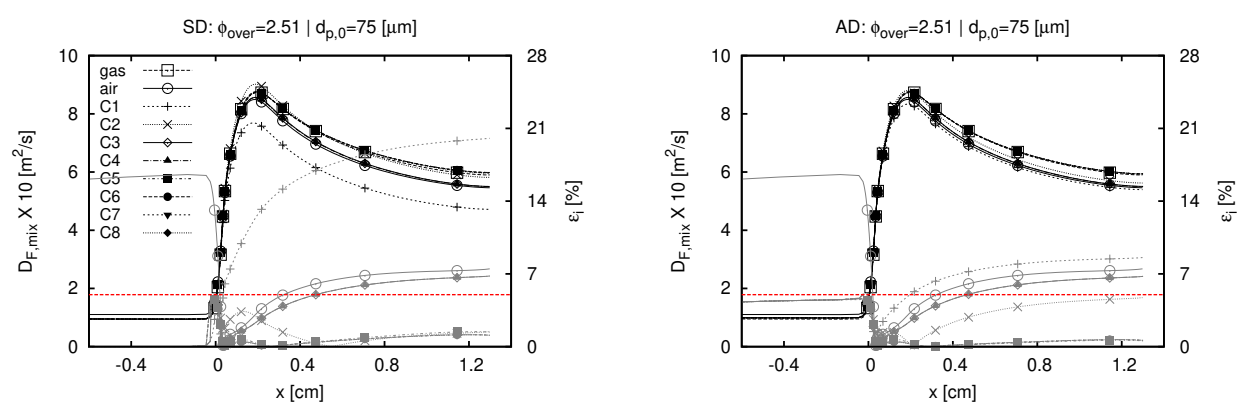

Figure 13: Binary diffusion coefficient $D_{\mathrm{F}, \mathrm{mix}}$ computations for the gas film throughout the droplet penetration for a rich mixture $\left(\phi_{\mathrm{over}}=2.51\right)$. Black $-D_{\mathrm{F} \text {,mix }}$, Gray $-\varepsilon_{i}$, Red - $5 \%$ deviation line.

in the pre-vaporization zone, where $\varepsilon_{\text {air }}$ reaches approximately $15 \%$. Initially unexpected, such deviations occur due to the computation of $D_{\mathrm{F} \text {,mix }}$ using the Fuller method (as presented in [28]). In our computations, this method is used for the mixture simplification by air. Since it differs from the method used to obtain $D_{\mathrm{F}, \text { mix }}$ from the chemical mechanism (see last part of section 3.1), deviations are found in the pre-vaporization region. Particularly, the determination of properties from the chemical mechanism is employed in the other computations presented in Fig. 13. This approach has been assumed in order to test the applicability of the Fuller method (as presented in [28]) in the present context, which is easier to implement in general CFD programs and also computationally less expensive as the one applied to the other combinations. As observed for the other properties, the increase of species number improves the estimation of $D_{\mathrm{F}, \mathrm{mix}}$. For $\mathrm{SD}$ calculations, $\mathrm{C} 5$ is the first combination to present deviations below $5 \%$ while $\mathrm{C} 2$ attends this condition in $\mathrm{AD}$ computations. However, by progressing to the more detailed combinations, $\mathrm{C} 3$ and $\mathrm{C} 4$ are back above the pre-defined threshold. Only from $\mathrm{C} 5$, the increase on the number of species stabilizes the deviation below $5 \%$.

In summary, the assessment addressed in this section shows that for some properties like $\mu$ and $D_{\mathrm{F}, \mathrm{mix}}$ the simplification of the actual mixture by air at same temperature and pressure is not so harmful. Nevertheless, specific heat and thermal conductivity values are strongly influenced by the mixture composition. In all scenarios, the combination of major species C5 attend the accuracy level required in this study for SD dilution methods. The same occurs for combination C6 in AD cases. Since the total number of species to be stored are smaller for C5 combined with SD when compared with $\mathrm{C} 6$ and $\mathrm{AD}$, we employ the former strategy in the further analysis.

\subsection{Validation of the simplification strategy on flames propagating in droplets mists}

Two of the major species combinations analyzed in the previous section are employed here to validate the proposed simplification strategy. The first is the combination C5 of the SD dilution method. The second is the combination C3 of the same dilution method. Despite showing deviations above 5\% for the evaporation properties, this option is analyzed due to the fact that its components (i.e. $\mathrm{CO}_{2}$ and $\mathrm{H}_{2} \mathrm{O}$ ) are frequently available in combustion chemistry simplifications. A variation of the combination $\mathrm{C} 5$ is also analyzed regarding the simplification of the actual mixture by air 
for $\mu$ and $D_{\mathrm{F}, \mathrm{mix}}$. One of the outcomes of the last section is that both properties are not so sensitive as $c_{p}$ and $\lambda$ to the mixture composition. Particularly, such a simplification for $D_{\mathrm{F}, \mathrm{mix}}$ is also motivated by computational issues, since the consideration of detailed mixture composition is more difficult to implement in a general CFD code and more expensive than its simplification as air. The combination assuming this simplification receives the suffix "-R"(from reduced) in its label, while the others "-F"(from full). The validation is also performed in terms of the speed of flames propagating in droplet mists here.

Figure 14 shows flame propagation speed results obtained for different $d_{p, 0}$ values, while keeping $\phi_{\text {over }}$ constant at 2.3. Only this overall rich mixture composition is evaluated here, since it demonstrated to be the most challenging scenario from Fig. 7. The three selected combinations effectively improve the representation of the actual mixture composition. The deviations seen in Fig. 7 of approximately 3.5\% for model $\mathrm{M} 2$ reduce at least seven times for C5-F and C5-R $(<0.45 \%)$, and three times for the combination $\mathrm{C} 3-\mathrm{F}(\approx 1.0 \%)$. Similarly, deviations of approximately $2 \%$ in Fig. 7 for model M1 reduce at least five times for C5-F and C5-R $(<0.35 \%)$, while a half for the combination $\mathrm{C} 3-\mathrm{F}(<0.9 \%)$. For both models, the $\mathrm{C} 5$ combinations are twice as effective than combination $\mathrm{C} 3$. However, both demonstrate to bring significant improvements for evaporation in combustion environment. Specifically, the simplification of the actual mixture by air for $\mu$ and $D_{\mathrm{F}, \text { mix }}$ is an advantageous simplification, since the computational time $e^{12}$ of $\mathrm{C} 5-\mathrm{R}$ is about $1 \%$ faster than that for $\mathrm{C} 5-\mathrm{F}$ and marginal differences are perceived between both approaches. It is important to highlight that estimations of the computational time are based on the present simulations. In case of the simulation of a real application spray flame, where the number of parcels can be larger than 1 million, the influence of the algorithm efficiency will be much more pronounced.
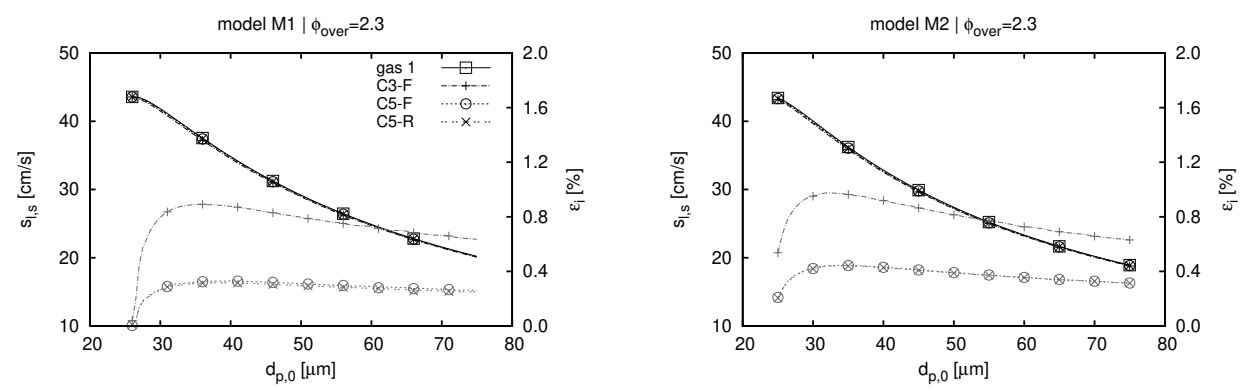

Figure 14: $s_{l, s}$ in function of $d_{p, 0}$ computed with evaporation mode M1 (left) and M2 (right) for a rich overall equivalence ratio. Black - $s_{l, s}$, Gray - deviation between simplification strategies and reference solution.

The improvements observed in Fig. 14 are also seen in Fig. 15, where variations

\footnotetext{
${ }^{12}$ The computational time has been based on the number of couplings between the Eulerian and the Lagrangian modules, using the same number of iterations. If only the algorithms used to address $\mu$ and $D_{\mathrm{F}, \text { mix }}$ are considered alone, differences in computational time should be more pronounced.
} 
in $\phi_{\text {over }}$ are investigated while keeping $d_{p, 0}=75 \mu \mathrm{m}$. Specifically, the observed gains in accuracy between Figs. 8 and 15 have in general the same proportionality as those noticed between Figs. 7 and 14 . The highest deviation seen in Fig. 8 of approximately $3.5 \%$ for model M2 reduces about seven times for C5-F and C5-R $(<0.45 \%)$, and three times for almost the entire range computed with the combination $\mathrm{C} 3-\mathrm{F}(\approx 1.0 \%)$. An exception occurs for mixture compositions richer than the one corresponding to the maximum value of $s_{l, s}$. Close to the upper flammability limits, the computations performed with the combination C3-F present higher values of $\varepsilon$ than those seen in Fig. 7 concerning the representation of the mixture as air. Yet the maximum deviation of approximately $2 \%$ in Fig. 8 for model M1 reduces at least five times for C5-F and C5-R $(<0.35 \%)$, and a half for the combination C3-F $(<0.9 \%)$. It is also important to highlight that, the $\phi_{\text {over }}$ value related to the maximum deviation is shifted when the simplification strategy of the mixture composition changes for both models. In Fig. 7 this maximum is found for $\phi_{\text {over }} \in[4.0,6.0]$, whereas in Fig. 14 it varies for $\phi_{\text {over }} \in[2.0,4.0]$ in C5 computations and clearly approaches to $\phi_{\text {over }}=4.0$ when the combination $\mathrm{C} 3-\mathrm{F}$ is used.

Differences between C5-F and C5-R are more pronounced in Fig. 15, however they are still fairly low. This outcome reinforces that the simplification of the actual mixture by air for $\mu$ and $D_{\mathrm{F}, \mathrm{mix}}$ is a valid approach.
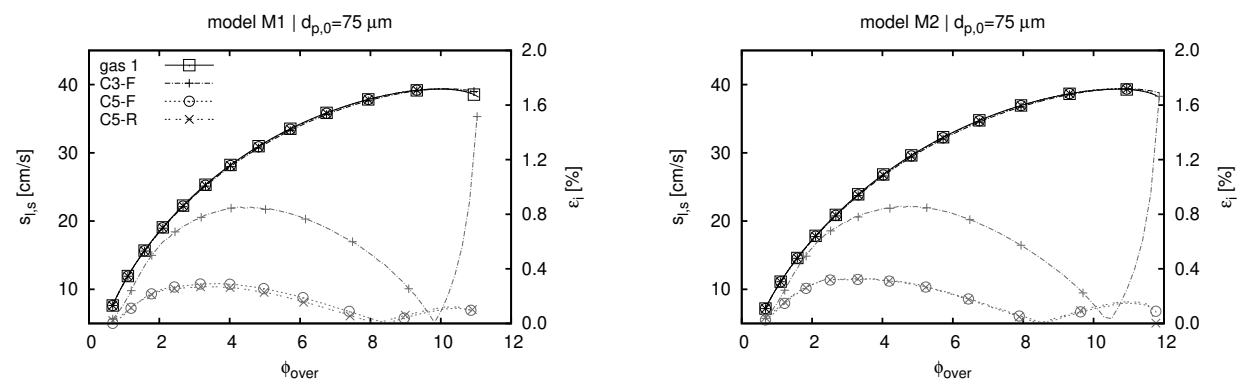

Figure 15: $s_{l, s}$ in function of $\phi_{\text {over }}$ computed with evaporation mode M1 (left) and M2 (right) for droplet mists of $d_{p, 0}=75 \mu \mathrm{m}$. Black - $s_{l, s}$, Gray - deviation between simplification strategies and reference solution.

The results presented in this section show that the proposed strategy is an accurate technique to simplify the actual mixture composition carrying evaporating droplets within reacting flows. Despite showing deviations above $5 \%$ for the evaporation properties, the combination $\mathrm{C} 3$ is also a profitable simplification for cases where only $\mathrm{CO}_{2}$ and $\mathrm{H}_{2} \mathrm{O}$ are available. However, when the usage of more species is feasible, more significant improvements are obtained with the inclusion of $\mathrm{CO}$ and $\mathrm{H}_{2}$ along with the combination C5. This conclusion applies to both investigated evaporation models.

\section{Summary and Conclusions}

Analyses of two droplet evaporation models have been conducted in single droplet environment and in flames propagating in homogeneous droplet mists. The study systematically evolves from non-reacting to reacting atmospheres. Different procedures 
used to estimate the vapor pressure on the droplet surface are evaluated in a single droplet context. Similarly, simplifications applied to the carrier gas phase are assessed in the sequence. These include simplifications in the diffusion transport of the vapor $(L e=1)$, as well as in the detailed composition of the mixture by pure air. For the former simplification, tests are conducted for different fuels in quiescent and forced convection environments. The influence of the two selected evaporation models and the simplification of the detailed composition of the mixture by air are subsequently analyzed for flames propagating in droplets mists. To reduce the deviations found by the mixture simplification, a new approach is proposed and successfully validated to represent this mixture in combustion environment.

Along with the single droplet simulations the model denoted as M2 demonstrates to be more universal than M1. It shows better performance for different operating conditions and liquid compositions as well. With respect to the different procedures used to address the vapor pressure, the Wagner equation (here labeled as VP 3) and Antoine equations (VP 4 and 5) agree quite well to each other. Considering that the Wagner equation covers a broad range of operating conditions (see Poling et al. [33]), this is therefore preferred here. The analysis of the unity Lewis number assumption just confirm the expected (see for instance [1]). Namely, the non-unity Le must be considered otherwise wrong evaporation rates are computed. The last part of single droplet investigations is dedicated to an artificial test about the influence of the detailed composition of the carrier gas. Different evaporation rates could be identified, which motivates analyses in combustion processes. From simulations of flames propagating in droplet mists, it is noticed that deviations between models are quite well pronounced and both are influenced by the simplification of the actual mixture by air. Particularly, model M2 is more sensitive than model M1 for this simplification. Deviations are quantified for different overall mixture compositions, variations in initial droplets sizes, and injection positions.

In view of the sensitivity of both models to the gas composition, a feasible approach for an accurate representation of the carrier gas thermo-chemical properties of droplets evaporating in combustion environment is proposed. Initially, a methodology is defined to determine the major species existing throughout the droplet penetration length. Afterwards, ten species combinations are investigated using the relevant properties to the evaporation modeling. The combination of $\mathrm{CO}_{2}, \mathrm{H}_{2} \mathrm{O}, \mathrm{CO}$, and $\mathrm{H}_{2}$ using the SD method (see section 3.4 reduces 7 and 3.5 times the deviations obtained respectively with models $\mathrm{M} 2$ and $\mathrm{M} 1$ in computations employing air properties. As a result, deviations are lower than $0.5 \%$ from cases computed with detailed mixture composition. Additional analysis shows that only $c_{p}$ and $\lambda$ are relevant properties to be described with more details, while $\mu$ and $D_{\text {F,mix }}$ are accurately characterized in terms of air. In cases where $\mathrm{CO}$ and $\mathrm{H}_{2}$ data are not available, the simple consideration of $\mathrm{CO}_{2}$ and $\mathrm{H}_{2} \mathrm{O}$ already reduces by a half deviations observed when computations are performed using air properties with both models.

It is important to highlight that, despite being conceived for reduced chemistry calculations, the proposed approach can be a useful strategy in detailed chemistry applications. Indeed, detailed information of the mixture composition is available in detailed chemistry computations, however the carrier mixture can be accurately and effectively described by the proposed strategy in order to alleviate computational demand. This is 
important when massive computation is involved, for instance on the construction of spray tabulated chemistry. In fact the computation of a single spray flamelet is not a computationally expensive task (see e.g. [5]). But when various scenarios must be covered to construct a valid range of representative states, many combinations of boundary and droplet injection conditions must be considered [9, 36-38]. Observe that in this context, simulations based on spray flamelets must have a consistent evaporation modeling with the procedure employed on the table generation, otherwise computed and tabulated thermo-chemical states will have weak correspondence.

\section{Acknowledgements}

We acknowledge the financial support from São Paulo Research Foundation (FAPESP

- grant \# 2017/06815-7). The Deutsche Forschungsgemeinschaft (DFG) is also acknowledged through the grant SFB/TRR 150.

\section{References}

[1] W. A. Sirignano, Fluid Dynamics and Transport of Droplets and Sprays, 2nd ed., Cambridge University Press, New York, NY, 2010.

[2] P. Jenny, D. J. E. M. Roekaerts, N. Beishuizen, Modeling of turbulent dilute spray combustion, Prog. Energy Combust. Sci. 38 (2012) 846-887.

[3] T. Kitano, J. Nishio, R. Kurose, S. Komori, Effects of ambient pressure, gas temperature and combustion reaction on droplet evaporation, Combust. Flame 161 (2014) 551-564.

[4] B. Franzelli, A. Vié, M. Boileau, B. Fiorina, N. Darabiha, Large Eddy Simulation of Swirled Spray Flame Using Detailed and Tabulated Chemical Descriptions, Flow, Turbul. Combust. (2016) 1-29.

[5] F. L. Sacomano Filho, N. Speelman, J. A. van Oijen, L. P. H. de Goey, A. Sadiki, J. Janicka, Numerical analyses of laminar flames propagating in droplet mists using detailed and tabulated chemistry, Combust. Theory Model. 22 (2018) 9981032 .

[6] E. Knudsen, Shashank, H. Pitsch, Modeling partially premixed combustion behavior in multiphase LES, Combust. Flame 162 (2015) 159-180.

[7] F. L. Sacomano Filho, G. Kuenne, M. Chrigui, A. Sadiki, J. Janicka, A consistent Artificially Thickened Flame approach for spray combustion using LES and the FGM chemistry reduction method: Validation in Lean Partially Pre-Vaporized flames, Combust. Flame 184 (2017) 68-89.

[8] F. L. Sacomano Filho, J. Kadavelil, M. Staufer, A. Sadiki, J. Janicka, Analysis of LES-based combustion models applied to an acetone turbulent spray flame, Combust. Sci. Technol. (2018) 1-14. 
[9] Y. Hu, H. Olguin, E. Gutheil, A spray flamelet/progress variable approach combined with a transported joint PDF model for turbulent spray flames, Combust. Theory Model. 21 (2017) 575-602.

[10] A. Both, RANS-FGM simulation of n-heptane spray flame in OpenFOAM: A new implementation of flamelet generated manifold to account for enthalpy loss with detailed reaction mechanisms, Master's thesis, Delft University of Technology, Delft, The Netherlands, 2017.

[11] M. Chrigui, F. Sacomano, A. Sadiki, A. R. Masri, Evaporation Modeling for Polydisperse Spray in Turbulent Flow, 2014, pp. 55-77.

[12] L. Ma, B. Naud, D. Roekaerts, Transported PDF Modeling of Ethanol Spray in Hot-Diluted Coflow Flame, Flow, Turbul. Combust. 96 (2016) 469-502.

[13] F. L. Sacomano Filho, Novel approach toward the consistent simulation of turbulent spray flames using tabulated chemistry, Phd thesis, Technische Universitaet Darmstadt, Darmstadt, Germany, 2017.

[14] D. Noh, S. Gallot-Lavallée, W. P. Jones, S. Navarro-Martinez, Comparison of droplet evaporation models for a turbulent, non-swirling jet flame with a polydisperse droplet distribution, Combust. Flame 194 (2018) 135-151.

[15] F. L. Sacomano Filho, G. C. Krieger Filho, J. A. van Oijen, A. Sadiki, Numerical Investigation Of Droplet Evaporation Modeling In Combustion Environment, in: ICLASS 2018 14th Int. Conf. Liq. At. Spray Syst., Chicago, 2018, p. 8.

[16] R. S. Miller, K. Harstad, J. Bellan, Evaluation of equlibrium and non-equlibrium evaporation models for many droplet gas liqud flow simulations, Int. J. Multiph. Flow 24 (1998) 1025-1055.

[17] S. S. Sazhin, Advanced models of fuel droplet heating and evaporation, Prog. Energy Combust. Sci. 32 (2006) 162-214.

[18] B. Abramzon, W. A. Sirignano, Droplet vaporization model for spray combustion calculations, Int. J. Heat Mass Transf. 32 (1989) 1605-1618.

[19] B. Rochette, E. Riber, B. Cuenot, Effect of non-zero relative velocity on the flame speed of two-phase laminar flames, Proc. Combust. Inst. (2018).

[20] Y. Hu, R. Kurose, Partially premixed flamelet in LES of acetone spray flames, Proc. Combust. Inst. (2018).

[21] P. Sierra Sanchez, Modeling the dispersion and evaporation of sprays in aeronautical combustion chambers, Phd thesis, Institut National Polytechnique de Toulouse - INPT, Toulouse, France, 2012.

[22] G. Kuenne, A. Ketelheun, J. Janicka, LES modeling of premixed combustion using a thickened flame approach coupled with FGM tabulated chemistry, Combust. Flame 158 (2011) 1750-1767. 
[23] N. Marinov, A detailed chemical kinetic model for high temperature ethanol oxidation, Int. J. Chem. Kinet. 31 (1999) 183-220.

[24] L. M. T. Somers, The simulation of flat flames with detailed and reduced chemical models, Ph.D. thesis, Technische Universiteit Eindhoven, 1994.

[25] A. Neophytou, E. Mastorakos, Simulations of laminar flame propagation in droplet mists, Combust. Flame 156 (2009) 1627-1640.

[26] M. C. Yuen, L. W. Chen, On Drag of Evaporating Liquid Droplets, Combust. Sci. Technol. 14 (1976) 147-154.

[27] K. Stephan, A. Laesecke, The Thermal Conductivity of Fluid Air, J. Phys. Chem. Ref. Data 14 (1985) 227-234.

[28] I. V. Gnielinski, VDI-Wärmeatlas, VDI Buch, Springer Berlin Heidelberg, Berlin, Heidelberg, 2006.

[29] E. W. Lemmon, R. T. Jacobsen, S. G. Penoncello, D. G. Friend, Thermodynamic Properties of Air and Mixtures of Nitrogen, Argon, Oxygen from $60 \mathrm{~K}$ to $2000 \mathrm{~K}$ at Pressures to 2000MPa, J. Phys. Chem. 29 (2000) 331.

[30] J. A. van Oijen, A. Donini, R. J. M. Bastiaans, J. H. M. ten Thije Boonkkamp, L. P. H. de Goey, State-of-the-art in premixed combustion modeling using flamelet generated manifolds, Prog. Energy Combust. Sci. 57 (2016) 30-74.

[31] S. B. Saharin, B. Lefort, C. Morin, C. Chauveau, L. Le Moyne, R. Kafafy, Vaporization characteristics of ethanol and 1-propanol droplets at high temperatures, At. Sprays 22 (2012) 207-226.

[32] S. B. Saharin, Vaporization and Autoignition Characteristics of Ethanol and 1propanol Droplets: Influence of Water, Phd thesis, University of Burgundy, Dijon (Bourgogne), France, 2013.

[33] B. Poling, J. M. Prausnitz, J. P. O'Connell, The properties of gases and liquids, 5th ed., McGRAW-HILL, 2001.

[34] C. G. Downingm, The evaporation of drops of pure liquids at elevated temperatures: Rates of evaporation and wet-bulb temperatures, AIChE J. 12 (1966) 760-766.

[35] S.-C. Wong, A.-C. Lin, Internal temperature distributions of droplets vaporizing in high-temperature convective flows, J. Fluid Mech. 237 (1992) 671.

[36] H. Olguin, E. Gutheil, Influence of evaporation on spray flamelet structures, Combust. Flame 161 (2014) 987-996.

[37] B. Franzelli, A. Vié, M. Ihme, On the generalisation of the mixture fraction to a monotonic mixing-describing variable for the flamelet formulation of spray flames, Combust. Theory Model. 19 (2015) 773-806. 
[38] B. T. Bojko, P. E. DesJardin, On the development and application of a droplet flamelet-generated manifold for use in two-phase turbulent combustion simulations, Combust. Flame 183 (2017) 50-65. 